\title{
Gesundheitsrisiko Mobilfunkstrahlung? Was ändert sich mit 5G?
}

\section{Health Risk Mobile Phone Radiation? What Will Change with 5G?}

(c) (ㄱ) ()

Autoren

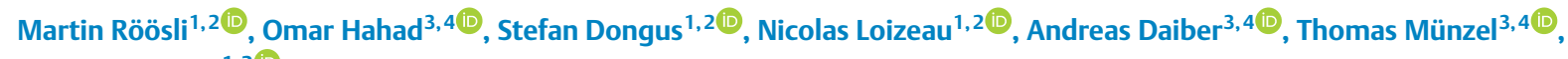
Marloes Eeftens ${ }^{1,2}$ (D)

Institute

1 Schweizerisches Tropen- und Public Health-Institut, Basel, Schweiz

2 Universität Basel, Basel, Schweiz

3 Zentrum für Kardiologie, Kardiologie I, Universitätsmedizin der Johannes Gutenberg-Universität Mainz, Mainz, Deutschland

4 Deutsches Zentrum für Herz-Kreislauf-Forschung (DZHK), Standort Rhein-Main, Mainz, Deutschland

Schlüsselwörter

5G, Mobilfunk, Tumor, elektromagnetische Hypersensibilität, Exposition

Key words

5G, mobile phone, tumour, electromagnetic hypersensitivity, exposure

\section{Bibliografie}

Aktuel Kardiol 2021; 10: 531-536

DOI 10.1055/a-1545-0875

ISSN 2193-5203

(C) 2021. The Author(s).

This is an open access article published by Thieme under the terms of the Creative Commons Attribution-NonDerivative-NonCommercial-License, permitting copying and reproduction so long as the original work is given appropriate credit. Contents may not be used for commercial purposes, or adapted, remixed, transformed or built upon. (https://creativecommons.org/licenses/by-nc-nd/4.0/).

Georg Thieme Verlag KG, Rüdigerstraße 14,

70469 Stuttgart, Germany

Korrespondenzadresse

Prof. Martin Röösli

Epidemiology and Public Health

Schweizerisches Tropen- und Public Health-Institut

P. O. Box

CH-4002 Basel, Schweiz

martin.roosli@swisstph.ch

\section{ZUSAMMENFASSUNG}

Die Exposition der Bevölkerung durch hochfrequente elektromagnetische Felder (HF-EMF) wird durch die körpernahe Nutzung von drahtlosen Kommunikationsgeräten dominiert. Die Exposition durch körperferne Sendeanlagen liegt im Durchschnitt mehrere Größenordnungen unterhalb der internationalen Richtwerte. Mit der zunehmenden mobilen Datennutzung und der damit verbundenen Nutzung von höheren Frequenzen für $5 \mathrm{G}$ ist mit einer Verdichtung des Mobilfunknetzes zu rechnen. Damit steigt aber nicht notgedrungen die HFEMF-Gesamtexposition der Bevölkerung, da Mobiltelefone bei besserer Signalqualität weniger stark emittieren. 5G ist eine technologische Weiterentwicklung der bisherigen Mobilfunktechnologie mit gleichen biophysikalischen Eigenschaften. Bisher konnten keine gesundheitlichen Auswirkungen unterhalb der Richtwerte konsistent nachgewiesen werden. Beobachtete biologische Effekte wie beispielsweise auf die elektrische Aktivität des Gehirns oder auf das oxidative Gleichgewicht bei hoher lokaler Exposition im Bereich der Expositionsrichtwerte stellen nach heutigem Kenntnisstand kein Gesundheitsrisiko dar.

\section{ABSTRACT}

Exposure of the population to radiofrequency electromagnetic fields (RF-EMF) is dominated by the use of wireless communication devices close to the body. Exposure from transmitters far from the body is on average several orders of magnitude below the international guideline values. With increasing mobile data usage and the associated use of higher frequencies for $5 \mathrm{G}$, a densification of the mobile network is to be expected. However, this will not necessarily increase the overall RF-EMF exposure of the population, as mobile phones emit less with better signal quality. $5 \mathrm{G}$ is a technological advancement of the previous mobile radio technology with the same biophysical properties. So far, no health effects below the guideline limits have been consistently demonstrated for RFEMF. Biological effects such as changes of the electrical activity of the brain or the oxidative balance were observed for high local exposure in the range of the exposure guideline limits. According to current knowledge, they do not represent a health risk. 


\section{Glossar}

HF-EMF hochfrequente elektromagnetische Felder

ICNIRP International Commission on Non-Ionizing Radiation Protection

ROS reaktive Sauerstoffspezies

SAR spezifische Absorptionsrate

\section{WAS IST WICHTIG?}

- Die Exposition der Bevölkerung gegenüber hochfrequenten elektromagnetischen Feldern (HF-EMF) wird hauptsächlich durch die eigene Nutzung von drahtlosen Kommunikationsgeräten bestimmt.

- $5 \mathrm{G}$ ist eine Weiterentwicklung der bestehenden Mobilfunktechnologie. Es gibt keine substanziellen Hinweise, dass $5 \mathrm{G}$ andere biologische Wirkungen hat als bisher verwendete Mobilfunktechnologien.

- Im Bereich der Grenzwerte für körpernah betriebene Geräte wurden biologische Auswirkungen beobachtet. Nach heutigem Kenntnisstand sind damit keine gesundheitlichen Risiken verbunden. Die Exposition durch Mobilfunkbasisstationen ist im Allgemeinen deutlich geringer als bei körpernah betriebenen Endgeräten, und damit auch eventuelle gesundheitliche Risiken.

\section{Grenzwerte für körpernahe und körperferne Quellen}

Drahtlose Kommunikationsgeräte emittieren hochfrequente elektromagnetische Felder (HF-EMF). Bei der Regulierung und Charakterisierung der HF-EMF-Exposition der Bevölkerung wird zwischen körpernah betriebenen Quellen (z. B. Mobil- und Schnurlostelefone, Laptops) und körperfernen HF-EMF-Quellen (z. B. Mobilfunkbasisstationen, Radio- und Fernsehantennen, Mobiltelefone anderer Personen) unterschieden. Bei körpernah betriebenen Geräten findet eine lokale Exposition statt und die spezifische Absorptionsrate (SAR), gemittelt über $10 \mathrm{Gramm}$ Gewebe, ist die maßgebliche Expositionsgröße (Einheit: W/kg). Unter Berücksichtigung eines Sicherheitsfaktors von 10 betragen die Expositionsrichtwerte der ICNIRP (International Commission on Non-Ionizing Radiation Protection) für lokale Absorption im Frequenzbereich von $100 \mathrm{kHz}$ bis $6 \mathrm{GHz}$ für die allgemeine Bevölkerung $2 \mathrm{~W} / \mathrm{kg}$ (Rumpf und Kopf) und $4 \mathrm{~W} / \mathrm{kg}$ (Gliedmaßen) [1]. Für berufliche Expositionen sind die Richtwerte jeweils 5-mal höher ( $\triangleright$ Abb. 1). Diese Grenzwerte gelten für alle europäischen Länder.

Bei körperfernen Quellen ist der ganze Körper einem mehr oder weniger homogenen Feld ausgesetzt, was mit der Ganzkörper-SAR charakterisiert wird. Der Richtwert für die Allgemeinbevölkerung beinhaltet einen Sicherheitsfaktor von 50 und beträgt $0,08 \mathrm{~W} / \mathrm{kg}$. Da die SAR nicht direkt messbar ist, wird für die Regulierung die Ganzkörper-SAR frequenzabhängig in eine elektrische Feldstärke (in $\mathrm{V} / \mathrm{m}$ ) oder in eine Leistungsflussdichte (in $\mathrm{W} / \mathrm{m}^{2}$ ) umgerechnet.
Die Expositionsrichtwerte der ICNIRP [1], welche die Grundlagen für die Festsetzung der Grenzwerte in europäischen Ländern bilden, liegen bei den gegenwärtig genutzten Mobilfunkfrequenzen zwischen 36 und $61 \mathrm{~V} / \mathrm{m}$. Oberhalb von $6 \mathrm{GHz}$ wird HF-EMF nur oberflächlich absorbiert und daher empfiehlt die ICNIRP als Richtwert für die Ganzkörperexposition eine Leistungsflussdichte von $10 \mathrm{~W} / \mathrm{m}^{2}$ (statt eine SAR) [1].

\section{KURZGEFASST}

Bei der Festlegung der Expositionsrichtwerte für körpernahe Endgeräte (z. B. Mobiltelefone) wurde ein Sicherheitsfaktor von 10 berücksichtigt und für körperferne Sender (z. B. Mobilfunkbasisstationen bzw. Sendemasten) ein Sicherheitsfaktor von 50. Für Letztere gelten also tiefere Grenzwerte.

\section{Exposition der Bevölkerung durch hochfrequente elektromagnetische Felder}

Persönliche Messungen mit tragbaren Geräten haben sich bewährt, um die typische Exposition gegenüber körperfernen Quellen im Alltag zu erfassen. In der EU-Studie GERONIMO wurden solche Messungen bei 529 Kindern im Alter von 8 bis 18 Jahren aus Dänemark, Holland, Schweiz, Slowenien und Spanien zwischen 2014 und 2016 durchgeführt. Die mittlere Exposition lag bei $0,17 \mathrm{~V} / \mathrm{m}$, also Größenordnungen tiefer als die Expositionsrichtwerte für körperferne Quellen. Ähnliche Werte wurden auch in anderen Ländern und bei Erwachsenen beobachtet [2]. Werte über $1 \mathrm{~V} / \mathrm{m}$ sind selten ( $\bullet$ Abb. 1). Beispielsweise lagen bei persönlichen Messungen von zufällig ausgewählten Personen aus dem Kanton Zürich (Schweiz) im Jahr 2015 weniger als 1\% aller Messwerte oberhalb von $1 \mathrm{~V} / \mathrm{m}$ [3]. Ein Wert von $1 \mathrm{~V} / \mathrm{m}$ entspricht einem Ganzkörper-SAR-Wert von etwa $0,01 \mathrm{~mW} / \mathrm{kg}$ und einer maximalen lokalen SAR von etwa $0,25 \mathrm{~mW} / \mathrm{kg}$ [1]. Bei einem Mobiltelefon können im ungünstigsten Fall rund 1000-mal stärkere lokale SARWerte auftreten $(2 \mathrm{~W} / \mathrm{kg})$.

Persönliche Messstudien erfassen die Strahlenabsorption durch körpernah betriebene Quellen nicht adäquat. Für kumulative Abschätzungen der HF-EMF-Absorption braucht es daher dosimetrische Berechnungen, wie sie in der EU-Studie GERONIMO für 1755 Personen aus Frankreich, Holland, Schweiz und Spanien anhand ihrer mobilen Nutzungsdaten durchgeführt wurden [4]. Die mittlere kumulative absorbierte Ganzkörper- bzw. Gehirndosis betrug $290 \mathrm{~mJ} / \mathrm{kg} / \mathrm{d}$ und $819 \mathrm{~mJ} / \mathrm{kg} / \mathrm{d}$. Wie aus Abb. 2 ersichtlich, sind Mobiltelefonanrufe die Hauptquelle für die Gehirndosis (83\%). Körperferne Quellen tragen nur 7,7\% zur kumulativen Gehirndosis bei (Mobilfunkbasisstationen: 4,1\%). Zur Ganzkörperdosis tragen ebenfalls hauptsächlich körpernahe Quellen bei (68\%). Neben Mobiltelefonanrufen sind der mobile Datenverkehr und die Nutzung von Tablets eine wichtige Ursache. Mobilfunkbasisstationen machen 8,3\% der Ganzkörperdosis aus und alle Fernfeldquellen zusammen $20 \%$. 


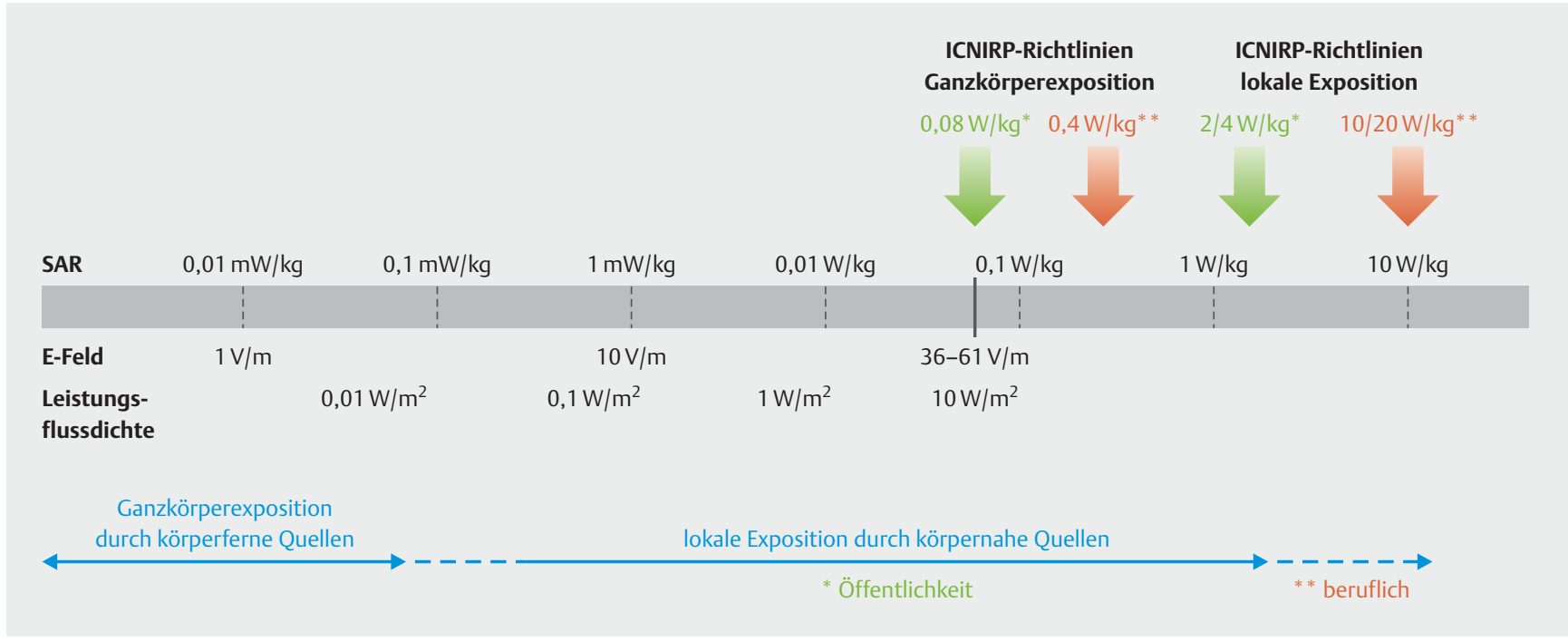

- Abb. 1 Überblick über die Einheiten, ICNIRP-Expositionsrichtwerte für HF-EMF im Frequenzbereich 100 kHz bis 6 GHz sowie typische Expositionswerte im Alltag durch körperferne und körpernahe Quellen. Die dargestellte Äquivalenz von SAR und E-Feld (elektrische Feldstärke in V/m) bzw. Leistungsflussdichte gilt näherungsweise für den Mobilfunkfrequenzbereich. ICNIRP: International Commission on Non-Ionizing Radiation Protection, SAR: spezifische Absorptionsrate

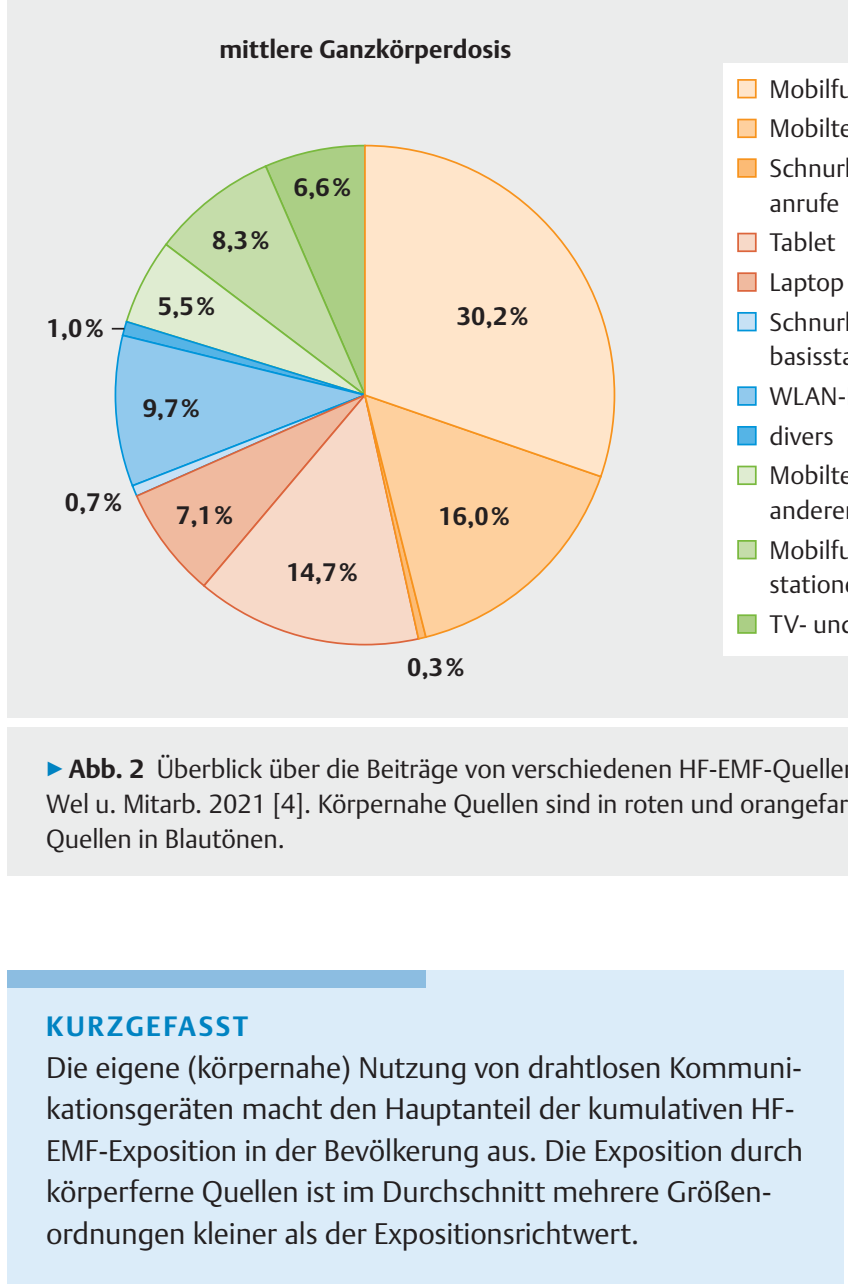

\section{Was ändert sich mit 5G?}

5G (New Radio) ist eine Weiterentwicklung der vorherigen Mobilfunkstandards 2G (GSM), 3G (UMTS) und 4G (LTE) mit dem Ziel, eine größere Datenrate zu erreichen, welche mit geringerer Verzögerung (Latenz) übertragen werden kann. Damit sollen neue Anwendungen möglich gemacht werden. Das Modulationsverfahren ist ähnlich wie bei 4G und WLAN (v. a. Orthogonal FrequencyDivision Multiplexing). 5G kann theoretisch auf allen bisherigen Mobilfunkfrequenzen betrieben werden. Zusätzlich werden im Vergleich zu den heutigen Mobilfunkfrequenzen sowohl tiefere 
Frequenzen um $700 \mathrm{MHz}$ - bisher für Fernsehsignalübertragungen genutzt - wie auch höhere Frequenzen um $3,5 \mathrm{GHz}$ eingesetzt (bisher WiMAX). In Zukunft ist geplant, für 5G auch das Frequenzspektrum oberhalb von $24 \mathrm{GHz}$ zu nutzen. Je höher die Frequenz, desto höher ist die Übertragungsdämpfung und desto geringer ist die Eindringtiefe in den Körper. So führt bei gleicher Sendeleistung ein Mobiltelefonat bei 3,5 GHz zu einer 6- bzw. 2-mal geringeren Strahlenabsorption des Gehirns im Vergleich zu den gegenwärtig genutzten Frequenzen um 1 bzw. $2 \mathrm{GHz}$ [5].

Wie sich die Einführung von $5 \mathrm{G}$ gesamthaft auf die Exposition der Bevölkerung auswirken wird, hängt von den zukünftigen Applikationen ab, die zurzeit noch weitgehend unbekannt sind. So war bei der Einführung von $2 \mathrm{G}$ auch nicht vorhersehbar, dass Textnachrichten eine wichtige Anwendung dieser Technologie sein würden. Danach schaffte der 3G-Standard die Voraussetzungen für die Nutzung von Smartphones, welche einige Jahre später entwickelt wurden.

Grundsätzlich führt, unabhängig von 5G, die zunehmende mobile Datennutzung zu einem erhöhten Bedarf an Mobilfunkbasisstationen. 5G ist effizienter als bisherige Mobilfunktechnologien, und damit nehmen die Emissionen pro übermittelte Datenmenge ab [6]. Eine weitere Neuerung von 5G sind adaptive Antennen. Das bedeutet, dass gezielt in die Richtung der Datennutzung gesendet wird. Je höher die Frequenz, desto gezielter kann die Senderichtung eingestellt werden. Bei den 5G-Frequenzen um $3,5 \mathrm{GHz}$ kann der Sendewinkel auf etwa $10^{\circ}$ reduziert werden, während konventionelle Antennen typischerweise einen Sendewinkel von 60 bis $120^{\circ}$ besitzen. Mit diesem sogenannten „Beamforming“ kann zeitlich begrenzt die Exposition am Ort von starker Datennutzung ansteigen. Jedoch nimmt sie in allen anderen Gebieten ab. Simulationsstudien kommen zum Schluss, dass die durchschnittliche Exposition bei adaptiven 5G-Antennen bei gleicher übermittelter Datenmenge rund 2- bis 5-mal geringer ist als mit den heutigen Technologien [5, 7]. Zudem sind die Emissionen von 5G-Mobilfunkbasisstationen im Stand-by-Betrieb geringer als bei älteren Technologien.

Die zunehmende mobile Datennutzung und die damit verbundene Nutzung von höheren Frequenzen für $5 \mathrm{G}$ wird zu einer Zunahme von Mobilfunkbasisstationen führen. Das muss aber nicht notwendigerweise eine Zunahme der Bevölkerungsexposition gegenüber HF-EMF nach sich ziehen. So kam eine Simulationsstudie für die Schweiz zum Schluss, dass mehr Mobilfunkbasisstationen, d.h. eine Reduktion des Zellenradius, zu einer Verringerung der Gesamtexposition von Mobilfunknutzenden um einen Faktor 2-10 führen [5]. Der Hauptgrund liegt darin, dass in einem dichteren Netz die Signalqualität besser ist und damit die Sendeleistung des eigenen Mobiltelefons geringer ausfällt. Je nach Art der Technologie und Art der Nutzung kann der Unterschied durch diese Leistungsregelung einen Faktor von 100000 oder mehr ausmachen [8].
KURZGEFASST

5G ist eine Weiterentwicklung der gegenwärtigen Mobilfunktechnologie mit praktisch gleichen biophysikalischen Eigenschaften. Aus Sicht der HF-EMF-Exposition bietet 5C eine Möglichkeit, die zunehmende mobile Datennutzung ganz oder zumindest teilweise mit höherer technologischer Effizienz zu kompensieren. Zudem dringen höhere Frequenzen weniger tief in den Körper ein.

\section{Bewertung der gesundheitlichen Risiken}

Unbestritten ist, dass HF-EMF eine thermische Wirkung haben. Beim Expositionsrichtwert der ICNIRP für die Allgemeinbevölkerung beträgt die maximale Erwärmung der Körperkerntemperatur bei Ganzkörperexposition $0,02{ }^{\circ} \mathrm{C}$ und die lokale Erwärmung $0,2^{\circ} \mathrm{C}$ (Kopf und Rumpf) bzw. $0,5^{\circ} \mathrm{C}$ (Gliedmaßen). Dies kann potenziell das Herz-Kreislauf-System beeinflussen. Ein niederländisches Expertengremium kommt jedoch, basierend auf 3 epidemiologischen Studien und 24 humanexperimentellen Studien, zum Schluss, dass HF-EMF unterhalb der Richtwerte keinen Einfluss auf das kardiovaskuläre und vegetative Nervensystem haben [9]. Mehr Evidenz gibt es zum Einfluss von hohen lokalen HF-EMFExpositionen im Bereich des Richtwerts auf die elektrische Aktivität des Gehirns im wachen Ruhezustand und im Schlaf [6]. In der Mehrzahl der randomisierten Humanstudien wurden dabei Veränderungen im Alpha-Frequenzbereich beobachtet, obwohl in einigen Studien kein Effekt gefunden wurde oder andere Frequenzbereiche betroffen waren. Die beobachteten Veränderungen liegen im Schwankungsbereich der normalen Werte. Sie wirkten sich nicht auf die kognitive Leistungsfähigkeit oder die subjektive Schlafqualität aus, und in der großen Mehrheit der Studien war die Makrostruktur des Schlafes, d.h. die Verteilung der Schlafphasen, nicht beeinflusst [6].

In In-vitro- und In-vivo-Studien wurden bei unterschiedlichsten Expositionsbedingungen Einflüsse von HF-EMF auf die Bildung von reaktiven Sauerstoffspezies (ROS) beobachtet [10]. Möglicherweise ist zumindest ein Teil dieser Resultate auf die thermische Wirkung von HF-EMF zurückzuführen, wie es auch für Infrarotstrahlung beobachtet wird. Die Produktion von ROS könnte theoretisch aber auch ein Indiz für längerfristige schädliche Auswirkungen sein. Epidemiologische Studien zu bösartigen Hirntumoren im Zusammenhang mit Mobiltelefonnutzung zeigen jedoch mehrheitlich kein erhöhtes Erkrankungsrisiko [11]. Vereinzelt beobachtete erhöhte Risiken in Fallkontrollstudien sind wahrscheinlich methodisch bedingt und auf eine systematische Überschätzung der retrospektiv erhobenen Telefonnutzungsdauer von Hirntumorpatienten im Vergleich zu gesunden Kontrollpersonen zurückzuführen. Da mittlerweile der größte Teil der Bevölkerung ein Mobiltelefon nutzt, müsste sich ein relevant erhöhtes Tumorrisiko in einem Anstieg der Hirntumorerkrankungen in den letzten Jahren geäußert haben. Zeittrendanalysen in mehreren Ländern ergaben jedoch keine Hinweise, dass die Inzidenz von Tumoren im Kopfbereich mit einer gewissen Verzögerung ansteigt, nachdem die Mehrheit der Bevölkerung in den entsprechenden Ländern begonnen hat, Mobiltelefone zu benutzen. Vereinzelte Beobachtungen 
von einer Zunahme der Glioblastome können plausibel auf eine Änderung in der diagnostischen Kodierungspraxis zurückgeführt werden, da gleichzeitig andere Arten von Hirntumoren abgenommen haben, jedoch die Gesamtzahl konstant blieb. In 4 Fallkontrollstudien aus Südkorea, Großbritannien, Deutschland und der Schweiz wurden keine konsistenten Zusammenhänge zwischen Kinderleukämien und HF-EMF-Exposition durch körperferne Quellen beobachtet [6].

Ein Teil der Bevölkerung führt Befindlichkeits- und Gesundheitsstörungen auf die Belastung durch elektromagnetische Felder in ihrem Wohn- oder Arbeitsumfeld zurück. Diese Selbstattribution, für welche es keine messbaren diagnostischen Kriterien gibt, wird als elektromagnetische Hypersensibilität bezeichnet. Eine Vielzahl von experimentellen Doppelblindstudien, die teilweise auch elektromagnetisch hypersensible Personen einschlossen, ergeben starke Evidenz gegen die Auslösung unspezifischer Beschwerden durch kurzfristige HF-EMF-Exposition [12]. Für langfristige HFEMF-Expositionen wurde in den meisten epidemiologischen Studien mit methodisch guter Expositionsabschätzung keine Beeinträchtigung des Wohlbefindens durch Exposition gegenüber körperfernen HF-EMF-Quellen am Wohnort beobachtet [6]. In Bezug auf körpernahe Mobiltelefonnutzung fand eine große prospektive Kohortenstudie aus Finnland und Schweden keine Zunahme von Kopfschmerzen [13] oder Schlafproblemen [14] innerhalb von 4 Jahren.

Bis vor Kurzem gab es keine qualitativ genügende epidemiologische Studie zum Einfluss der Mobilfunkstrahlung auf die Spermienqualität, obwohl das Thema öffentlich kontrovers diskutiert wird. In einer kürzlich veröffentlichten prospektiven Kohortenstudie wurde bei rund 3000 Personen kein Einfluss von einem Mobiltelefon in der vorderen Hosentasche auf die Spermienqualität und die Zeitdauer bis zum Nachweis einer Schwangerschaft beobachtet [15].

Zusammenfassend lässt sich feststellen, dass unterhalb der Expositionsrichtwerte keine gesundheitlichen Auswirkungen konsistent nachgewiesen sind. In Bezug auf die zukünftige Nutzung von höheren Frequenzen, die auf der Körperoberfläche absorbiert werden, sind mögliche Auswirkungen auf die Haut und das Auge noch vertieft zu untersuchen, auch wenn es keine substanziellen Hinweise gibt, dass dieser Frequenzbereich, welcher zwischen den gegenwärtig genutzten Mobilfunkfrequenzen und Infrarotstrahlung liegt, andere, noch unbekannte biophysikalische Auswirkungen hätte.

\section{Fazit}

Unterhalb der Expositionsrichtwerte konnten bisher keine gesundheitlichen Auswirkungen konsistent nachgewiesen werden. Biologische Auswirkungen im Bereich des Richtwerts für körpernah betriebene HF-EMF-Quellen stellen gemäß heutigem Kenntnisstand kein Gesundheitsrisiko dar. Es gibt keine fundierten Hinweise, dass $5 \mathrm{G}$ andere gesundheitliche Auswirkungen als die bisherigen drahtlosen Kommunikationstechnologien hat.
Interessenkonflikt

Die Forschung von Martin Röösli ist vollständig von öffentlichen oder gemeinnützigen Stiftungen finanziert. Martin Röösli ist und war als Berater für eine Reihe von nationalen und internationalen öffentlichen Beratungs- und Forschungslenkungsgruppen bezüglich der möglichen gesundheitlichen Auswirkungen der Exposition gegenüber nichtionisierender Strahlung tätig, darunter die Weltgesundheitsorganisation, die International Agency for Research on Cancer (IARC), die Internationale Kommission zum Schutz vor nichtionisierender Strahlung (ICNIRP), die Schweiz (Mitglied der Arbeitsgruppe "Mobilfunk und Strahlung" und Vorsitzender der Expertengruppe BERENIS), die deutsche Strahlenschutzkommission (Mitglied des Ausschusses Nichtionisierende Strahlung (A6) und Mitglied der Arbeitsgruppe 5C (A630)) und die unabhängige Expertengruppe der schwedischen Strahlenschutzbehörde. Von 2011 bis 2018 war M.R. unbezahltes Mitglied des Stiftungsrates der Schweizerischen Forschungsstiftung Strom und Mobilkommunikation, einer gemeinnützigen Forschungsstiftung an der ETH Zürich. Weder Industrie noch Nichtregierungsorganisationen sind im wissenschaftlichen Beirat der Stiftung vertreten.

Literatur

[1] ICNIRP. Guidelines for Limiting Exposure to Electromagnetic Fields (100 kHz to $300 \mathrm{GHz}$ ). Health Phys 2020; 118: 483-524. doi:10.1097/HP. 0000000000001210

[2] Jalilian $\mathrm{H}$, Eeftens $\mathrm{M}$, Ziaei $\mathrm{M}$ et al. Public exposure to radiofrequency electromagnetic fields in everyday microenvironments: An updated systematic review for Europe. Environ Res 2019; 176: 108517. doi:10.1016/ j.envres.2019.05.048

[3] Röösli M, Struchen B, Eeftens $M$ et al. Persönliche Messungen von hochfrequenten elektromagnetischen Feldern bei einer Bevölkerungsstichprobe im Kanton Zürich 2016. Im Internet (Stand: 03.10.2021): https:// www.zh.ch/de/umwelt-tiere/luft-strahlung/mobilfunk.html\#1966350537

[4] van Wel L, Liorni I, Huss A et al. Radio-frequency electromagnetic field exposure and contribution of sources in the general population: an organ-specific integrative exposure assessment. J Expo Sci Environ Epidemiol 2021. doi:10.1038/s41370-021-00287-8

[5] Kuehn S, Pfeifer S, Kuster N. Total Local Dose in Hypothetical 5 G Mobile Networks for Varied Topologies and User Scenarios. Appl Sci 2020; 10: 5971. doi:10.3390/app10175971

[6] UVEK. Bericht Mobilfunk und Strahlung. Bern: UVEK; 2019. Im Internet (Stand: 29.10.2021): https://www.bafu.admin.ch/bafu/de/home/themen/elektrosmog/mitteilungen.msg-id-77294.html

[7] Matalatala M, Deruyck M, Shikhantsov S et al. Multi-Objective Optimization of Massive MIMO $5 \mathrm{G}$ Wireless Networks towards Power Consumption, Uplink and Downlink Exposure. Appl Sci 2019; 9: 4974. doi:10.339 0/app9224974

[8] Mazloum T, Aerts S, Joseph W et al. RF-EMF exposure induced by mobile phones operating in LTE small cells in two different urban cities. Ann Telecommun 2019; 74: 35-42. doi:10.1007/s12243-018-0680-1

[9] Health Council of the Netherlands. Background Document to the advisory Report 5 G and Health. The Hague: Health Council of the Netherlands; 2020.

[10] Schuermann D, Mevissen M. Manmade Electromagnetic Fields and Oxidative Stress-Biological Effects and Consequences for Health. Int J Mol Sci 2021; 22: 3772. doi:10.3390/ijms22073772

[11] Röösli M, Lagorio S, Schoemaker MJ et al. Brain and Salivary Gland Tumors and Mobile Phone Use: Evaluating the Evidence from Various Epidemiological Study Designs. Annu Rev Public Health 2019; 40: 221-238. doi:10.1146/annurev-publhealth-040218-044037 
[12] Schmiedchen K, Driessen S, Oftedal G. Methodological limitations in experimental studies on symptom development in individuals with idiopathic environmental intolerance attributed to electromagnetic fields (IEI-EMF) - a systematic review. Environ Health 2019; 18: 88. doi:10.118 6/s12940-019-0519-x

[13] Auvinen A, Feychting M, Ahlbom A et al. Headache, tinnitus and hearing loss in the international Cohort Study of Mobile Phone Use and Health (COSMOS) in Sweden and Finland. Int J Epidemiol 2019; 48: 1567-1579. doi:10.1093/ije/dyz127
[14] Tettamanti G, Auvinen A, Akerstedt T et al. Long-term effect of mobile phone use on sleep quality: Results from the cohort study of mobile phone use and health (COSMOS). Environ Int 2020; 140: 105687. doi:10. 1016/j.envint.2020.105687

[15] Hatch EE, Willis SK, Wesselink AK et al. Male cellular telephone exposure, fecundability, and semen quality: results from two preconception cohort studies. Hum Reprod 2021; 36: 1395-1404. doi:10.1093/humrep/deab0 01 